\title{
Global-local Structural Optimization Using Response Surfaces of Local Optimization Margins
}

Boyang Liu (boyang@aero.ufl.edu) and Raphael T. Haftka (haftka@ufl.edu)

Department of Mechanical and Aerospace Engineering, University of Florida, Gainesville, FL 32611-6250

Layne T. Watson (1tw@ cayuga.cs.vt.edu)

Department of Computer Science

Virginia Polytechnic Institute and State University, Blacksburg, VA 24061

\begin{abstract}
A general decomposition method developed by the authors is applied to global-local structural optimization problems. First, a large number of component optimizations for maximization of margins are performed. Response surface approximations (RSA) for maximum margins of component optimization are constructed. At the system level optimization, the RSA of maximum margins are used as surrogate for the components. One advantage of the decomposition approach is that it allows much of the search for a global optimum to be conducted in low-dimensions for each component separately. Minimization of a portal frame weight with eight local optima is used to demonstrate the approach.
\end{abstract}

Key Words decomposition, constraint margins, local/global structural optimization, response surfaces

\section{Introduction}

For the design of realistic structures carrying a large number of loading cases and having many components that need design variables to describe detailed geometry, direct single-level optimization often requires of exorbitant computer resources. The computational resources required for the solution of an optimization problem typically increase with dimensionality of the problem at a rate that is more than linear. This is particularly true when global optima are sought, as the rate of increase in cost with the number of variables is usually exponential. One obvious solution is to break up a large optimization problem into smaller sub-problems and a coordination problem to preserve the couplings among these sub-problems.

Decomposition usually allows easy utilization of parallel computation capabilities. Moreover, to break a big problem into several small problems is natural in engineering optimization, because engineers tend to work in teams, each concentrating on parts of a project. This allows a broad work front and shortens development time.

Numerous hierarchical and nonhierarchical decomposition strategies for the optimization of large scale systems, comprised of interacting subsystems, have been proposed — Schmit et al.'s $(1978,1982)$ multilevel structural decomposition, Sobieski's concurrent subspace optimization (CSSO), (1988 ) nonhierarchical decomposition, Kroo's collaborative optimization (CO) (1996), etc. However, most of these strategies may not always converge to the optimum of the original problem (e.g, Alexandrov and Lewis, 2000) 
There is a class of quasi-separable optimization problems narrow enough to allow rigorous decomposition theory, yet general enough to encompass many large scale engineering problems. The subsystems for these problems involve local design variables and global system variables, but no variables from other subsystems. The objective function is a sum of a global system criterion and the subsystem's criteria.

Each subsystem is given a budget and global system variable values, and then asked to independently maximize its constraint margins. For each subsystem, a response surface approximation of maximum constraint margins is constructed as a function of the global design variables, and it is used as a surrogate for the overall optimization. A special version of this approach was developed for coordinating panel design with overall wing design by Liu et al. (2000). Haftka et al. (2002) developed general theory for global optimization with continuous variables. The objective of the present paper is to demonstrate the applicability of the method to decomposition of structural optimization based on structural components.

The methodology is demonstrated for design of a simple portal frame structure that has three rail-cross-section beams.

\section{Decomposition of Single-level Optimization and Response Surfaces of Maximum Subsystem Margins}

Consider a system with a vector of system design variables $s\left(s=\left\{s_{i}, \underline{s}_{2}, \ldots, \underline{s}_{N}\right\}\right)$. The system is composed of $M$ subsystems, each with its own vector of subsystem local variables $\boldsymbol{l}_{\underline{i}}, j=1 \ldots, M,\left(\boldsymbol{l}_{\underline{i}}=\left\{l_{j 1}, \ldots . ., l_{j k}\right\}\right)$. The single-level optimization of the system has the following form:

$$
\begin{aligned}
& \underset{s, l}{\min \operatorname{imize}_{s,}} W(s) \\
& \text { subject to } \mathrm{g}_{\mathrm{q}}(s) \leq 0, \mathrm{q}=1, \cdots, \mathrm{Q} \\
& \qquad \mathrm{g}_{\mathrm{kp}}\left(s, 1_{j}\right) \leq 0, \mathrm{k}=1, \cdots, \mathrm{M}, \mathrm{p}=1, \cdots, \mathrm{P}
\end{aligned}
$$

where $\mathrm{W}$ is the system objective function, $\boldsymbol{g}_{\boldsymbol{q}}$ are system-level constraints which only depend on the system design variables, $\boldsymbol{g}_{k p}$ are constraints which depend on the system design variables and design variables of the $\boldsymbol{k}_{\boldsymbol{t} \boldsymbol{h}}$ subsystem.

The authors have proposed a two-level decomposition of the optimization problem ( Haftka et al., 2002), where each subsystem maximizes the constraint margin for its constraints. That is the upper-level problem is defined as

$$
\begin{aligned}
\underset{s .}{\min \operatorname{imize}_{\text {e }}} & W(s) \\
\text { subject to } & \mathrm{g}_{\mathrm{q}}(s) \leq 0, \mathrm{q}=1, \cdots, \mathrm{Q} \\
& -\mu_{\mathrm{j}}(s) \leq 0, \mathrm{j}=1, \cdots, \mathrm{M}
\end{aligned}
$$

where $\mu_{j}$ is the minimum margin of the constraints in the $j$ th subsystem, which is maximized at the lower level. 
The maximization of the minimum margin $\mu_{j}$ is a typical min-max problem, with the known difficulty in that the maximum of a function does not have continuous derivatives. Following Taylor and Bendsøe (1984), we solve this problem by introducing the margin $\mu_{\mathrm{j}}$ as an additional design variable. The subsystem optimization is then stated with given system variables $s$ as follows

$$
\begin{aligned}
& \underset{l_{j}}{\max \operatorname{imize}_{j}} \mu_{j} \\
& \text { subject to } \mathrm{g}_{\mathrm{jp}}\left(s, l_{j}\right)+\mu_{j} \leq 0, \quad \mathrm{p}=1, \cdots, \mathrm{P}
\end{aligned}
$$

We have shown ( Haftka et al., 2002) that under mild conditions, every local optimum of the decomposed system is also a local optimum of the original system, so that the decomposition does not introduce spurious solutions. In addition, the decomposition permits us to perform some global optimization at the subsystem level. Because the cost of global optimization can grow exponentially with problem size, we gain by performing much of the global search at the subsystem level.

Because subsystem optima are not smooth functions of system level design variables, gradient-based optimization may experience difficulties with the two-level formulation. In addition, the implementation of a two-level scheme may require difficult integration of component optimization software. Response surface approximations (RSA) offer an attractive way of overcoming both implementation and smoothness difficulties.

First, a large number of subsystem level optimizations for different values of the global design variables are performed. Then, the results of the subsystem optima are fitted with a simple response surface, typically a lower order polynomial. Finally, the system level optimization is performed with the RSA as a surrogate for the maximum margin of each subsystem.

\section{Frame Example}

A portal frame, shown in Fig.1, has been used often to demonstrate decomposition strategies (e.g., Sobieski et al., 1985). The structure consists of three beams. Each beam has a rail-section defined by six size design variables defined in Fig. 2. The portal frame is subjected to a point load and a concentrated moment. Material properties are given in Table 1.

\section{Table 1 Material Properties}

\begin{tabular}{|c|c|}
\hline $\boldsymbol{E}$ & $7.06 \mathrm{E6} \mathrm{N} / \mathrm{cm}^{2}$ \\
\hline$v$ & 0.33 \\
\hline$\sigma_{\boldsymbol{y}}$ & $\pm 20000 \mathrm{~N} / \mathrm{cm}^{2}$ \\
\hline$\tau$ & $11600 \mathrm{~N} / \mathrm{cm}^{2}$ \\
\hline
\end{tabular}



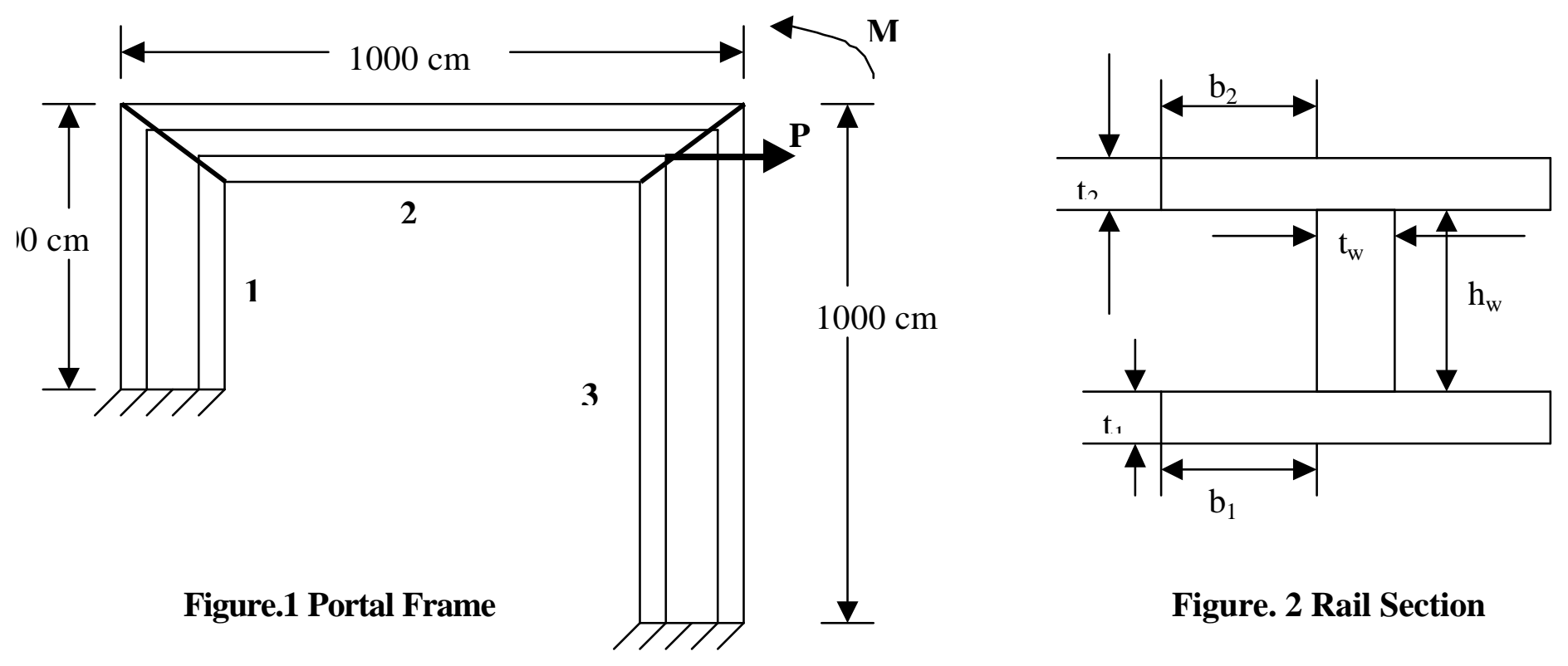

Figure. 2 Rail Section

In order to demonstrate the advantage of searching for optima at the local level, the problem has been modified to create multiple distinct local optima. This was accomplished by requiring that the top and bottom flange of each beam be different in area by at least $10 \%$. This type of constraints may be realistic when it is important to assist the assembly process of a system by reducing possible confusion as to questions like "which side is up?". These constraints divide the design space into eight disjoint feasible regions or islands. In each one of these islands, a particular choice of the larger flange for each beam holds. We will use $\mathrm{T}$ to denote that the top flange is bigger and $\mathrm{B}$ to denote that the bottom flange is larger. So TTB corresponds to a design with the top flange being larger for the first two beams and the bottom flange being bigger for the third beam.

\subsection{Problem formulation}

Each rail-section beam has six variables $b_{1}, t_{1}, b_{2}, t_{2}, h_{w}, t_{w}$ as shown in Fig. 2, for a total of 18 design variables. Three types of constraints are considered in the optimization. Stress constraints include flange normal stress and web shear stress constraints. Geometric constraints limit the aspect ratios and area distributions to eliminate extremely slender plates that are likely to buckle, Finally, the flange area constraint, requiring that top flange area is $10 \%$ more than bottom flange area, or bottom flange area is $10 \%$ more than top flange area is used to create multiple local optima. Objective function is portal frame volume.

The optimization problem is formulated as

$$
\text { Minimize } W=\sum A_{i} l_{i}
$$

where $A_{i}, l_{i}, i=1, \ldots 3$, are section areas and length of the beams.

Subject to

$$
g_{i, j}=\frac{\sigma_{i, j}}{\sigma_{y}}-1 \leq 0 \quad \text { (flange normal stress), } i=1,2,3, j=1,2,3,4 \text { ( } 4 \text { point normal }
$$




$$
\begin{gathered}
g_{i}=\frac{\tau_{i}}{\tau_{y}}-1 \leq 0 \quad(\text { web shear stress) } \quad, \mathrm{i}=1,2,3 \\
g_{i}=1-\frac{h_{w, i} t_{w, i}}{0.2\left(\left(2 b_{1, i}+t_{w, i}\right) t_{w, i}+h_{w, i} t_{w, i}+\left(2 b_{2, i}+t_{w, i}\right) t_{2, i}\right)} \leq 0, \mathrm{i}=1,2,3
\end{gathered}
$$

(web area constraints) $g_{l, i}=\frac{2 b_{l, i}+t_{w, i}}{20 t_{l, i}}-1 \leq 0,1=1,2, \mathrm{i}=1,2,3 \quad$ (top and bottom flange length is not more than 20 times of its thickness) $g_{i}=\frac{h_{w, i}}{35 t_{w, i}}-1 \leq 0, \mathrm{i}=1,2,3 \quad$ ( web depth is not more than 35 times of its thickness)

$$
g_{i}=1-\frac{\left(2 b_{2, i}+t_{w, i}\right) b_{2, i}}{1.1\left(2 b_{1, i}+t_{w, i}\right) t_{1, i}} \leq 0
$$

or

$$
g_{i}=1-\frac{\left(2 b_{1, i}+t_{w, i}\right) b_{1, i}}{1.1\left(2 b_{2, i}+t_{w, i}\right) t_{2, i}} \leq 0
$$

side constraints

$$
\begin{aligned}
& \mathrm{b}_{1, \min } \leq \mathrm{b}_{1, \mathrm{i}} \leq \mathrm{b}_{1, \text { max }} \\
& \mathrm{t}_{1, \min } \leq \mathrm{t}_{1, \mathrm{i}} \leq \mathrm{t}_{1, \max } \\
& \mathrm{b}_{2, \min } \leq \mathrm{b}_{2, \mathrm{i}} \leq \mathrm{b}_{2, \max } \\
& \mathrm{t}_{2, \min } \leq \mathrm{t}_{2, \mathrm{i}} \leq \mathrm{t}_{2, \max } \\
& \mathrm{h}_{\mathrm{w}, \min } \leq \mathrm{h}_{\mathrm{w}, \mathrm{i}} \leq \mathrm{h}_{\mathrm{w}, \max } \\
& \mathrm{t}_{\mathrm{w}, \min } \leq \mathrm{t}_{\mathrm{w}, \mathrm{i}} \leq \mathrm{t}_{\mathrm{w}, \text { max }}
\end{aligned}
$$

Constraint (7) requires that the web area is at least $20 \%$ of the total area of the beam, constraints (8) and (9) are aspect ratio limits, and (10) is the separation constraint.

For a given bending moment $\boldsymbol{M}$ and axial force $\boldsymbol{N}$ at one end, maximum normal stresses $\sigma_{i, j}$ at the top and the bottom flanges are calculated. Average web shear stress $\tau_{i}$ is calculated for given shear force $\boldsymbol{Q}$. For a given moment $\boldsymbol{M}_{\boldsymbol{A}}$ and shear force $\boldsymbol{Q}_{\boldsymbol{A}}$, at the end $\mathrm{A}$, bending moment of the other end $\mathrm{B}$ is calculated as follows:

$$
M_{B}=M_{A}+l Q_{A}
$$

where $l$ is length of the bar.

\subsection{Two-level Optimization}

For the two-level approach, the areas and moments of inertia of the beams are the system-level design variables. Furthermore, an approximate relationship between the area and moment of inertia was used to eliminate the moments of inertia. Using finite element analysis, the upper-level variables can be used to calculate the bending moments, axial loads and shear forces for each beam.

At the lower-level optimization, the minimum margin of all constraints is maximized for a given area, moment of inertia, bending moment, axial force and shear 
force, which are obtained from the areas and moments of inertia. Design variables are rail-section size variables, $b_{1}, t_{1}, b_{2}, t_{2}, h_{w}, t_{w}$, shown in Fig. 2 . Constraints are added to force the area and moment of inertia associated with the lower level variables to agree with the system level variables. Thus the lower level problem becomes:

\section{Maximize $\mu$}

Subject to (5)-(11), with the constraint relaxed by a margin $\mu$, so that for example (9) is replaced by

$$
g_{10}=\frac{h_{w}}{35 t_{w}}-1+\mu \leq 0
$$

and in addition the a prescribed area and moment of inertia must be matched

$$
\begin{aligned}
& g_{11}=\frac{A_{\text {local }}}{A_{g}}-1=0 \\
& g_{12}=\frac{I_{\text {local }}}{I_{g}}-1=0 \\
& I_{\text {Local }}=\frac{\left(2 b_{1}+t_{w}\right)^{3} t_{1}+\left(2 b_{2}+t_{w}\right)^{3} t_{2}+h_{w} t_{w}^{3}}{12}
\end{aligned}
$$

where $\boldsymbol{A}_{g}$ and $\boldsymbol{I}_{g}$ are area and moment of inertia from the upper-level optimization, $\boldsymbol{A}_{\text {local }}$ $\boldsymbol{I}_{\text {local }}$ are subsystem area and moment of inertia which are updated in the local-level optimization because of change of six rail-section size variables.

\section{2.1 Response Surface of Maximum Margins}

Cubic polynomial response surface approximations are used to obtain an approximate relationship between the maximum margin and the given area, moment of inertia, and loads. For creating the response surface, the JMP software (SAS 1995) was used to select a D-optimal set of points and fit the response surface. For better conditioning, the variables are normalized to the range $[-1,1]$. For example, the normalized area is given as

$$
\bar{A}=\frac{2 A-A_{\max }-A_{\min }}{A_{\max }-A_{\min }}
$$

with similar expression for the other variables.

where in the above expressions, "max" and " min" denote the ranges of variables.

The maximum margin $\mu^{*}$ is then expressed as

$$
\mu^{\bullet}=\mu^{\bullet}(\bar{A}, \bar{I}, \bar{M}, \bar{F}, \bar{Q})
$$

Ranges of three beams are as follows:

Beam No. 1

$$
\begin{aligned}
& 56 \mathrm{~cm}^{2}<\mathrm{A}<71 \mathrm{~cm}^{2} \\
& 9542 \mathrm{~cm}^{4}<\mathrm{I}<13207 \mathrm{~cm}^{4} \\
& (-) 12500000 \mathrm{~N} . \mathrm{cm}<\mathrm{M}<(-) 14000000 \mathrm{Ncm}
\end{aligned}
$$


$30000 \mathrm{~N}<\mathrm{F}<35000 \mathrm{~N}$

(-) $5000 \mathrm{~N}<\mathrm{Q}<(-) 5500 \mathrm{~N}$

Beam No. 2

$$
\begin{aligned}
& 68 \mathrm{~cm}^{2}<\mathrm{A}<95 \mathrm{~cm}^{2} \\
& 14033 \mathrm{~cm}^{4}<\mathrm{I}<22494 \mathrm{~cm}^{4} \\
& (-) 11500000 \mathrm{~N} . \mathrm{cm}<\mathrm{M}<(-) 11800000 \mathrm{Ncm} \\
& 50000 \mathrm{~N}<\mathrm{F}<55000 \mathrm{~N} \\
& 30000 \mathrm{~N}<\mathrm{Q}<35000 \mathrm{~N}
\end{aligned}
$$

Beam No. 3

$$
\begin{aligned}
& 29 \mathrm{~cm}^{2}<\mathrm{A}<43 \mathrm{~cm}^{2} \\
& 1435 \mathrm{~cm}^{4}<\mathrm{I}<2427 \mathrm{~cm}^{4} \\
& 900000 \mathrm{~N} . \mathrm{cm}<\mathrm{M}<1300000 \mathrm{Ncm} \\
& (-) 3100 \mathrm{~N}<\mathrm{F}<(-) 30000 \mathrm{~N} \\
& 1100 \mathrm{~N}<\mathrm{Q}<1600 \mathrm{~N} \\
& \text { “_ “ in (19) } 20(21) \text { represents direction of bending moment. Ranges of axial forces, }
\end{aligned}
$$
bending moment and shear forces in the above equations are estimated from finite element analysis of the portal structure. Maximum of all minimum margins is a very complicated function of the above five parameters. In order to obtain highly accurate margin RS, small ranges of five parameters are used here. In case that there are big ranges, it is suggested to break a big range into several small segments and in each segment, one RS will be fitted.

More than 16,000 design points were randomly generated for each of three beam ranges defined in (21)-(23), and then 150 D-optimal points were selected from each domain. The lower-level optimization was performed twice to for the two option of the separation constraint, and the best design of the two was selected, thus performing part of the global optimization at the local level. The resulting margin was fitted as a cubic polynomial. Table 3 shows the statistics of the response surfaces, including the coefficient of multiple determination, $\boldsymbol{R}$, and its adjusted value $\boldsymbol{R}_{\boldsymbol{a}}$. The table indicates good accuracy of the fit.

Table 2: Statistics Results of Three Margin Response Surfaces

\begin{tabular}{|c|c|c|c|}
\hline Statistics & Beam 1 & Beam 2 & Beam 3 \\
\hline$R^{2}$ & 0.9981 & 0.9989 & 0.9884 \\
\hline$R_{a}$ & 0.9970 & 0.9982 & 0.9816 \\
\hline Root Mean Square Error & 0.0036 & 0.0054 & 0.0115 \\
\hline Mean of Margin & -0.0048 & -0.0514 & 0.1502 \\
\hline RMS Error/ Mean (\%) & 75.00 & -10.51 & 7.66 \\
\hline
\end{tabular}

\subsubsection{Uppe r-level optimization}

In the upper-level optimization, the volume is minimized subject to the approximate margin constraint. GENESIS ( Vanderplaats Research and Development, 2000) is used to perform the upper-level optimization subject to three margin constraints 
for the three beams The SQP (sequence quadratic programming) optimization method is used, so that the system-level optimization is local. The moment of inertia $I$ of each beam is approximated in terms of its cross-sectional area $A$ as follows:

$$
I=\beta A^{\alpha}
$$

where $\beta$ is obtained by least square fit for a range of values of $\alpha$. The data for the fit included 69 rail cross-sections, which are created in terms of combinations of six size variables shown in Fig 2 within reasonable limits. As shown in Table 3, the average error in the approximation was small.

Table 3: Average Error in Approximation for Moment of Inertia

\begin{tabular}{|c|c|c|c|}
\hline Beam \# & $\beta$ & $\alpha$ & Average Error (\%) \\
\hline Beam 1 & 22.30 & 1.5 & $2.58 \%$ \\
\hline Beam 2 & 24.76 & 1.5 & $2.28 \%$ \\
\hline Beam 3 & 8.78 & 1.5 & $5.31 \%$ \\
\hline
\end{tabular}

The upper-level problem is then formulated as:

Design variables $A_{i}, i=1,2,3$

$$
\begin{aligned}
& \text { Minimize } W=\sum A_{i} l_{i} \\
& \text { Subject to } \mu_{i} \cdot\left(\bar{A}_{i}, \bar{I}_{i}, \bar{M}_{i}, \bar{N}_{i}, \bar{Q}_{i}\right) \geq 0, \mathrm{i}=1,2,3 \\
& \qquad A_{i, \min } \leq A_{i} \leq A_{i, \max }, \mathrm{i}=1,2,3
\end{aligned}
$$

where $\boldsymbol{l}_{\boldsymbol{i}}, \boldsymbol{\mu}_{i}^{\bullet}$ is the approximated maximum margin, and $A_{i, \min }, A_{i, \max }$ are the lower and upper bounds, respectively, for the area.

After the upper level optimum is found, the lower-level optimization needs to be repeated with the optimum values of the areas and moments of inertia. For this final component optimization, we first maximize the margin without the equality constraint for the moment of inertia in order to find a moment of inertia more matched for the final area. Next, a finite element analysis of the portal frame is performed to update loads for each beam. Then the margin is maximized with equality of constraints for area and moment of inertia. This process may still leave small violation of the constraints. It is possible to construct a more accurate response surface near the optimum, but we found that small increases in the areas and a repetition of the process described in this paragraph were sufficient to eliminate the violations.

\subsection{Results}

The optimum design had a larger top flange for all three beams. The third beam was mostly designed by minimum size constraints, and so the separation constraints were not important. The results are summarized in Table 4 . They show differences of up to $4 \%$ in the objective function between the eight designs. For the two-level optimization, the component optimization of first two beams led to the top flange being selected, so that the global search was completed at the local level. For the two-level approach, the response surface approximations resulted in a design with some constraint violations, so that the design was adjusted by a small increase in the areas of the three beams. The 
volume shown in Table 5 is still below the global optimum, reflecting some very small constraint violations.

Table 4. Comparison of Single -level and Two-level Optimal Areas $\left(\mathrm{cm}^{2}\right)$ and Volumes $\left(\mathrm{cm}^{3}\right)$

\begin{tabular}{|c|c|c|c|c|c|}
\hline & & Beam 1 & Beam 2 & Beam 3 & Volume \\
\hline \multirow{8}{*}{$\begin{array}{c}\text { Single- } \\
\text { level }\end{array}$} & 1 & 63.3 & 78.6 & 30.4 & 140630 \\
\hline & 2 & 63.3 & 78.6 & 30.5 & 140630 \\
\hline & 3 & 62.8 & 81.4 & 30.4 & 146150 \\
\hline & 4 & 62.8 & 81.4 & 30.4 & 143240 \\
\hline & 5 & 65.3 & 78.5 & 30.4 & 141570 \\
\hline & 6 & 65.3 & 78.5 & 30.4 & 141570 \\
\hline & 7 & 64.9 & 81.4 & 30.5 & 144180 \\
\hline & 8 & 64.9 & 81.3 & 30.5 & 144180 \\
\hline \multirow{2}{*}{ Two-level } & Two-level & 63.2 & 77.2 & 30.1 & 138900 \\
\hline & $\begin{array}{c}\text { Two-level } \\
\text { Adjusted }\end{array}$ & 63.2 & 78.2 & 30.5 & 140300 \\
\hline
\end{tabular}

The areas and volumes of the single-level and two-level designs are shown in Table 4 above. Table 5 shows the design variables for each beam and their margins from RSA and the lower-level optimization. We can see that margins from the lower-level optimization are off by about $2 \%$ for the first two beams, but there is a $10 \%$ violation for beam No. 3. The large approximation error for beam No. 3 is partly due to margin RSA errors and partly due to moment of inertia RSA errors. The area and local design variables reached their lower bounds for Beam 3, and this led to big approximation error.

In order to remedy the constraint violations the areas of beams No. 2 and No. 3 were increased by about one percent (see Table 5), and the process of component optimization was repeated. The results are shown in Table 6. It is seen that now the constraint violation are negligible.

The example demonstrates that even though the system optimization was local, the global optimum was found due to the global search at the component level (since both version of the separation constraint were considered). 
Table 5: Comparison of Margins from Local Optimization with Margins from RS

\begin{tabular}{|c|c|c|c|c|c|c|c|c|c|}
\hline $\begin{array}{c}\text { Beam } \\
\#\end{array}$ & $\begin{array}{c}\text { Flange } \\
\text { Constrain }\end{array}$ & \multicolumn{6}{|c|}{ Optimal Design of Rail Section Size } & $\begin{array}{c}\text { Margin } \\
\text { (Local) }\end{array}$ & $\begin{array}{c}\text { Margin } \\
\text { (RS) }\end{array}$ \\
\hline & & $\mathrm{b}_{1}$ & $\mathrm{t}_{1}$ & $\mathrm{~b}_{2}$ & $\mathrm{t}_{2}$ & $\mathrm{~h}_{\mathrm{w}}$ & $\mathrm{t}_{\mathrm{w}}$ & & \\
\hline \multirow{2}{*}{1} & $\mathrm{~T}$ & 7.32 & 1.15 & 7.01 & 1.32 & 30.24 & 0.86 & -0.0102 & \multirow{2}{*}{-0.0004} \\
\cline { 2 - 11 } & $\mathrm{B}$ & 6.50 & 1.43 & 9.37 & 0.95 & 30.03 & 0.83 & -0.0357 & \\
\hline \multirow{2}{*}{2} & $\mathrm{~T}$ & 9.89 & 1.02 & 10.57 & 1.08 & 23.88 & 0.95 & -0.0200 & \multirow{2}{*}{-0.0001} \\
\cline { 2 - 10 } & $\mathrm{B}$ & 6.50 & 1.79 & 6.98 & 1.61 & 32.54 & 0.88 & -0.0587 & \\
\hline \multirow{2}{*}{3} & $\mathrm{~T}$ & 6.50 & 0.75 & 6.50 & 0.75 & 15.00 & 0.64 & -0.1003 & \multirow{2}{*}{0.0012} \\
\cline { 2 - 9 } & $\mathrm{B}$ & 6.50 & 0.75 & 6.50 & 0.75 & 15.00 & 0.64 & -0.1003 & \\
\hline
\end{tabular}

T: Top flange area is $10 \%$ greater than bottom flange area

B: Bottom flange area is $10 \%$ greater than top flange area

Table 6: Maximum Margins for Adjusted Optima

\begin{tabular}{|c|c|c|c|c|c|c|c|c|}
\hline \multirow{2}{*}{$\begin{array}{c}\text { Beam } \\
\#\end{array}$} & \multirow{2}{*}{$\begin{array}{c}\text { Flange } \\
\text { Constrain }\end{array}$} & \multicolumn{6}{|c|}{ Optimal Design of Rail Section Size } & \multirow{2}{*}{$\begin{array}{l}\text { Margin } \\
\text { (Local) }\end{array}$} \\
\hline & & $b_{1}$ & $\mathrm{t}_{1}$ & $\mathrm{~B}_{2}$ & $\mathrm{t}_{2}$ & $\mathrm{~h}_{\mathrm{w}}$ & $t_{w}$ & \\
\hline \multirow{2}{*}{1} & $\mathrm{~T}$ & 6.50 & 1.11 & 6.94 & 1.17 & 32.63 & 0.93 & -0.0011 \\
\hline & B & 6.50 & 1.27 & 8.70 & 0.89 & 32.34 & 0.90 & -0.0231 \\
\hline \multirow{2}{*}{2} & $\mathrm{~T}$ & 6.67 & 1.31 & 6.92 & 1.46 & 36.46 & 1.04 & -0.0035 \\
\hline & B & 6.50 & 1.58 & 6.88 & 1.41 & 35.71 & 0.98 & -0.0357 \\
\hline \multirow{2}{*}{3} & $\mathrm{~T}$ & 6.50 & 0.75 & 6.94 & 0.78 & 15.00 & 0.60 & 0.0098 \\
\hline & B & 6.94 & 0.78 & 6.50 & 0.75 & 15.00 & 0.60 & 0.0098 \\
\hline
\end{tabular}

\section{Concluding Remarks}

A decomposition strategy for quasi-separable optimization problem was specialized to structural optimization. At the structural component level, the minimum margin of all constraints was maximized for given system level variables. Response surface approximation of maximum margin for the components was constructed by performing many component optimizations and integrated into the system optimization. The approach allows the search for a global optimum to proceed mostly at the component level. A three-beam frame example was used to demonstrate the approach. A constraint requiring $10 \%$ difference between the flanges of each beam created eight distinct local optima. The two-level approach identified the global optimum, even though at the system level local optimization was used. 


\section{Acknowledgement}

This research was supported by Air Force Office of Scientific Research Grant F4962099-1-0026. The authors highly appreciate Juan Pablo Leiva and Vanderplaats Research and Development for providing a GENESIS license.

\section{References}

Alexandrov, N. M., and Lewis, R. M., "Analytical and computational aspects of collaborative optimization “, NASA/TM-2000-210104, April, 2000.

Haftka, R.T., Watson L.T., and Liu, B., "Multidisciplinary design optimization with quasiseparable subsystems," submitted to Optimization in Engineering, 2002.

JMP Statistics and Graphics Guide: SAS Institute Inc., Cary, N.C. 1995.

Kroo, I., " Decomposition and collaborative optimization for large-scale aerospace design". In Multidisciplinary Design Optimization: State of the Art, SIAM, September, 1995.

Liu, B., Haftka, R.T., and Akgün, M.A., " Two-level composite wing structural optimization using response surfaces, "Structural Optimization, 20(2), pp.87 -96, 2000.

Liu. B. and Haftka, R.T., "Single-level composite wing optimization based on ?exural lamination parameters", submitted to Journal of Structural Optimization, 2002.

Schmit, L.A., and Ramannathan, R.K., “ Multilevel approach to minimum weight design including buckling constraints", AIAA J., 16(2), 1978, pp.97-104.

Schmit, L.A., and Mehrinfar, M.," Multilevel optimum design of structures with fibercomposite stiffened panel components", AIAA J., 20(1), 1982, pp. 138-147.

Sobieszczanski-Sobieski, J., " Optimization by decomposition : a Step from hierarchic to non-Hierarchic systems." Presented at the Second NASA/Air Force Symposium on Recent Advances in Multidisciplinary Analysis and Optimization (Held in Hampton), NASA CP3031, Part 1, 1988.

Sobieszczanski-Sobieski, J., James, B.B., and Dovi, A.R., "Structural optimization by multilevel decomposition, AIAA J. 23(11), 1985, pp. 1775-1782.

Taylor, J.E., and Bensøe, M.P., “ An Interpretation for Min-Max structural design problems including a method for relaxing constraints", International Journal of Solids and Structures, 30, 4, pp.301-314, 1984

Vanderplaats Research and Development, Inc., GENESIS User Manual, Version 6.0, January 2000, Colorado Spring, CO80906 